\title{
Indícios da integralidade do cuidado na prática da equipe de enfermagem na atenção paliativa oncológica
}

Evidence of care comprehensiveness in the practice of nursing staff in oncology palliative care

Indicios de integralidad del cuidado en práctica del equipo de enfermería en atención paliativa oncológica

Marcelle Miranda da Silva ${ }^{1}$, Marléa Chagas Moreira ${ }^{2}$, Joséte Luzia Leite ${ }^{3}$, Alacoque Lorenzini Erdmann ${ }^{4}$

${ }^{1}$ Enfermeira, Doutora em Enfermagem. Professora Adjunta da Escola de Enfermagem Anna Nery da Universidade Federal do Rio de Janeiro (EEAN/UFRJ). Rio de Janeiro, RJ, Brasil. E-mail: marcellemsufrj@gmail.com.

2 Enfermeira, Doutora em Enfermagem. Professora Associada da EEAN/UFRJ. Rio de Janeiro, RJ, Brasil. E-mail: marleachagas@gmail.com.

${ }^{3}$ Enfermeira. Doutora em Enfermagem. Professora Titular Emérita da Universidade do Estado do Rio de Janeiro. Rio de Janeiro, RJ, Brasil. E-mail: joluzia@gmail.com.

${ }^{4}$ Enfermeira. Doutora em Filosofia da Enfermagem. Professora Titular do Departamento de Enfermagem da Universidade Federal de Santa Catarina.

Florianópolis, SC, Brasil. E-mail: alacoque@newsite.com.br.

\section{RESUMO}

Objetivou-se identificar os indícios da integralidade do cuidado na prática dos profissionais da equipe de enfermagem no contexto da atenção paliativa oncológica. Estudo descritivo, qualitativo. Foram entrevistados sete enfermeiros e quatro técnicos de enfermagem. Utilizou-se a análise temática, evidenciando-se duas unidades temáticas: Indícios da integralidade do cuidado na prática dos profissionais da equipe de enfermagem; e $O$ trabalho em equipe com premissa da integralidade do cuidado. As ações da equipe de enfermagem sustentam-se na comunicação, convergindo esforços e formando rede com os demais profissionais para o atendimento das múltiplas necessidades da pessoa e seus familiares, dessa maneira, visando ao conforto e à qualidade de vida. Deve-se incentivar a autovalorização e autopercepção dos integrantes da equipe de enfermagem como elementos-chave na dinâmica e organização do trabalho, sendo primordial a disponibilidade de recursos para aplicação dos instrumentos de interação e a política organizacional que apoie a interdisciplinaridade e, por conseguinte, a integralidade.

Descritores: Cuidados Paliativos; Assistência Integral à Saúde; Enfermagem Oncológica.

\section{ABSTRACT}

The objective was to identify evidences of comprehensive care in the professional practice of the nursing staff in the context of oncology palliative care. In this descriptive, qualitative study, seven nurses and four nursing technicians were interviewed. Thematic analysis resulted two thematic units: Evidences of comprehensive care in the practice of the nursing professionals and teamwork as the premise of comprehensive care. The nursing staff's actions are based on communication, converging efforts, and forming a network with other professionals to meet the multiple needs of the individuals and their families in order to offer comfort and quality of life. Self-worth and self-perception of the nursing team should be encouraged as key elements in the dynamics and organization of the work, while the availability of resources is primordial to be able to apply the instruments of interaction and the organizational policy that supports being interdisciplinary and therefore complete and comprehensive.

Descriptors: Palliative Care; Comprehensive Health Care; Oncology Nursing.

\section{RESUMEN}

Se objetivó identificar indicios de integralidad del cuidado en la práctica de profesionales del equipo de enfermería actuantes en los puestos de atención paliativa oncológica. Estudio descriptivo, cualitativo. Se utilizó análisis temático, evidenciándose dos unidades temáticas: Indicios de integralidad del cuidado en práctica de profesionales del equipo de enfermería; y El trabajo en equipo con la premisa de integralidad del cuidado. Las acciones del equipo se sustentan en la comunicación, convergiendo esfuerzos y tejiendo red con los demás profesionales para la atención de las múltiples necesidades de la persona y familiares, buscando comodidad y calidad de vida. Debe incentivarse la autovaloración y autopercepción de los integrantes del equipo de enfermería como elementos clave en la dinámica y organización del trabajo, siendo primordial la disponibilidad de recursos para aplicación de los instrumentos de interacción y la política organizacional que apoye la interdisciplinaridad y, consiguientemente, la integralidad.

Descriptores: Cuidados Paliativos; Atención Integral de Salud; Enfermería Oncológica. 


\section{INTRODUÇÃO}

A integralidade, no Brasil, passou a fazer parte das propostas de reforma no campo da saúde no início da década de 1980, culminando como um dos princípios fundamentais do Sistema Único de Saúde (SUS), em 1990, na Lei $\mathrm{n}^{\circ} 8.080^{(1)}$. Compreende uma temática extensamente discutida em todos os contextos de atenção à saúde das pessoas, como no caso da atenção paliativa nas situações de ameaça à vida por doença, como por exemplo, o câncer.

A integralidade é dotada de diversos sentidos ${ }^{(2)}$. Em geral, tais sentidos abrangem diferentes noções. Três destas compreendem: a que incide sobre as práticas dos profissionais da área da saúde, considerando o valor do foco na pessoa, que é atendida de forma sistêmica. A segunda noção abrange a organização do trabalho nos serviços de saúde, de modo a formar redes entre os níveis de assistência à saúde e ampliar as possibilidades de apreensão das necessidades de saúde de um grupo populacional. Tais noções encaminham para a prática interdisciplinar, o que aumenta as possibilidades de aplicação da integralidade, não baseada apenas na postura de um único profissional, mas sim no senso do trabalho em equipe. A terceira, porém, não última noção existente, está ligada às políticas, conjugando os atributos das respostas governamentais a certos problemas de saúde, o que origina os programas, que visam atender as necessidades de grupos específicos ${ }^{(3)}$.

No presente estudo, a integralidade é entendida na lógica da prática profissional que considera o ser humano como um ser complexo, dotado de múltiplas dimensões ${ }^{(4-}$ 5). Nesta perspectiva, os fins da integralidade estão centrados no atendimento das necessidades da pessoa de forma contextualizada, conjugando o físico, o psicológico, o emocional, o espiritual e o social. Esta abordagem vai ao encontro do que preconiza a Organização Mundial da Saúde (OMS) na prática da atenção paliativa, sendo uma abordagem que objetiva promover a qualidade de vida às pessoas e seus familiares que enfrentam juntos os problemas associados com doenças que põem em risco a vida. Abrange a família como unidade de cuidado, valoriza essas múltiplas dimensões que podem estar envolvidas no processo de morrer, na prevenção e alívio do sofrimento, a partir do empenho dos profissionais da área da saúde ${ }^{(6)}$.

Assim, a integralidade na atenção paliativa, especialmente na oncologia, tem sido tratada na literatura a partir da valorização do atendimento de todas as necessidades da pessoa e seus familiares, por meio do trabalho em equipe, na perspectiva da interdisciplinaridade e da comunicação eficaz, focando a qualidade de vida na morte em detrimento da quantidade em dias de vida(7-9).

Apesar das diferentes noções envolvidas nos sentidos do termo integralidade, destaca-se que todas são importantes e precisam estar articuladas estrategicamente. Desse modo, uma noção não exclui a outra. Todas unem esforços e valores que defendem uma sociedade mais justa e solidária(3). Os desafios para a concretização da integralidade são grandes, uma vez que representa, em todas as noções apresentadas, uma luta contra o reducionismo e a fragmentação ${ }^{(4)}$.

A prática profissional e o senso de trabalho em equipe em prol da integralidade do cuidado foram abordados, neste estudo, a partir da perspectiva de profissionais da equipe de enfermagem que atuam no setor da internação hospitalar de uma unidade especializada na atenção paliativa oncológica. Neste contexto, esta modalidade de atendimento representa uma alternativa comum para controle de sintomas que se exacerbam na condição da doença oncológica avançada. Em muitas situações, a pessoa pode vir a falecer no decorrer deste período ${ }^{(6)}$. Destaca-se o valor da compreensão do fenômeno na perspectiva da prática dos profissionais de enfermagem, já que estão presentes em tempo integral e em maior número nas enfermarias. Logo, angariam subsídios para identificação e direcionamento do atendimento das necessidades da pessoa, bem como da família.

Em assim sendo, questiona-se: quais os indícios da integralidade do cuidado na prática dos profissionais da equipe de enfermagem que atuam nas enfermarias no contexto da atenção paliativa oncológica?

Objetivou-se identificar os indícios da integralidade do cuidado na prática dos profissionais da equipe de enfermagem que atuam nas enfermarias no contexto da atenção paliativa oncológica.

O estudo justifica-se diante da necessidade de discussão do tema relacionado à situação dos cuidados ao fim da vida no Brasil, que precisam ser incrementados, a partir da preservação da integralidade do cuidado. De acordo com a revisão de literatura identificou-se que a integralidade surge mediante princípio deste modo de cuidar, contudo, requer novas investigações diante dos desafios para a maior divulgação e oferta da atenção paliativa com base neste elemento em decorrência das múltiplas necessidades da população na contemporaneidade ${ }^{(10-12)}$. 


\section{MÉTODO}

Estudo do tipo descritivo, qualitativo, realizado em um Hospital especializado na atenção paliativa oncológica, localizado no município do Rio de Janeiro, Brasil.

Os dados foram coletados nas enfermarias do referido Hospital. São ao todo quatro enfermarias, com 14 leitos cada. Há um enfermeiro diarista em cada andar, porém, no momento da coleta de dados um dos andares estava desprovido deste profissional. Além dos enfermeiros diaristas, há dois enfermeiros plantonistas, que fazem escala de 12 por 60 horas, com complementação de carga horária para cumprimento de 40 horas semanais, e são os responsáveis por todos os andares no turno da noite, nos finais de semana e feriados. O setor conta com uma enfermeira chefe. O quantitativo de técnicos de enfermagem por andar varia de dois a quatro, dependendo da escala das complementações, no cumprimento de 40 horas semanais.

Participaram do estudo sete enfermeiros e quatro técnicos de enfermagem. Todos respeitaram os critérios de inclusão: ter vínculo empregatício com a Instituição e estar atuando na enfermaria por no mínimo seis meses. Foram excluídos os profissionais que estavam de férias, licenças ou que não foram encontrados no período da coleta de dados. A interrupção da coleta de dados se deu com a saturação dos mesmos, a partir do momento em que os depoimentos não trouxeram novas informações relevantes relacionadas à temática investigada, conforme orientação da pesquisa com abordagem qualitativa ${ }^{(13)}$.

A coleta de dados aconteceu de outubro de 2010 a fevereiro de 2011. A técnica utilizada foi a entrevista semiestruturada, permitindo maior flexibilidade para esclarecimento de pontos essenciais no conhecimento da realidade. As entrevistas foram conduzidas pelo roteiro: como você desenvolve o seu trabalho visando o atendimento das necessidades da pessoa hospitalizada e do seu familiar? Como você se vê inserido na equipe de enfermagem/de saúde?

Foi realizada a caracterização do perfil socioprofissional dos membros da equipe de enfermagem das enfermarias, de modo a facilitar a captação dos participantes do estudo. Esta caracterização foi viabilizada por meio do preenchimento pela própria pesquisadora de um instrumento contendo as seguintes variáveis: idade, sexo, tempo de formação profissional, tempo de atuação na oncologia, e, no caso dos enfermeiros, se possuem ou não pós-graduação na área.

Cada entrevista, realizada individualmente, foi gravada em dispositivo eletrônico de áudio, e transcrita na íntegra. Para preservação do anonimato dos participantes do estudo as entrevistas foram identificadas pela letra $E$ de enfermeiro e TE de técnico de enfermagem, seguidas do número de ordem de ocorrência das mesmas.

A análise dos dados foi realizada seguindo o método da Análise Temática. Esta é estruturada pelas seguintes etapas: pré-análise; exploração do material; tratamento dos resultados obtidos e interpretação(13-14). Assim, o processo analítico foi guiado pela identificação das principais percepções identificadas nas falas dos participantes do estudo, de acordo com os elementos que surgiram mais frequentemente, caracterizando os temas, que foram base para construção das unidades temáticas. Neste processo foram geradas duas unidades temáticas, a saber: indícios da integralidade do cuidado na prática dos profissionais da equipe de enfermagem; e o trabalho em equipe com premissa da integralidade do cuidado.

A discussão dos dados foi realizada a partir das contribuições dos preceitos da atenção paliativa e dos autores que discutem a temática na área de conhecimento da enfermagem.

Todos os participantes do estudo assinaram o Termo de Consentimento Livre e Esclarecido respeitando os aspectos éticos da Resolução do Conselho Nacional de Saúde $n^{\circ}$ 196/96. O estudo foi aprovado no Comitê de Ética em Pesquisa do Instituto Nacional de Câncer (INCA), com parecer no 45/2010.

\section{RESULTADOS}

$\mathrm{Na}$ caracterização do perfil dos sete enfermeiros, três eram diaristas, um plantonista diurno e três plantonistas noturnos; seis do sexo feminino; três na faixa etária entre 20 e 30 anos de idade, dois na faixa etária entre 31 e 40 anos, e outros dois entre 41 e 50 anos. Quanto ao tempo de formação profissional, em sua maioria, relacionado ao tempo de trabalho na oncologia, um enfermeiro apresentou três anos, dois apresentaram quatro anos, um seis anos, um 11 anos, outro 20 anos, e o último 28 anos de graduado. Apenas um enfermeiro não possuía título de pós-graduação, e cinco realizaram a Residência em Enfermagem em Oncologia no INCA.

$\mathrm{Na}$ caracterização do perfil dos quatro técnicos de enfermagem, três eram plantonistas diurnos e um plantonista noturno; dois do sexo feminino; um na faixa etária entre 20 e 30 anos de idade, outro na faixa entre 31 e 40 anos, outro entre 41 e 50 anos, e o último na faixa etária entre 51 e 60 anos. Quanto ao tempo de 
formação profissional, um informou possuir sete anos, outro oito anos, outro 18 anos e o último 35 anos. É válido ressaltar que dois técnicos de enfermagem informaram possuir curso de graduação completo em enfermagem. Um dos técnicos trabalha há 15 anos na Instituição, dois trabalham há quatro anos e um há três.

\section{Indícios da integralidade do cuidado na prática dos profissionais da equipe de enfermagem}

Nesta unidade temática foram retratados os indícios que favorecem a integralidade do cuidado nas ações diárias da equipe de enfermagem em prol do atendimento das necessidades da pessoa hospitalizada com câncer avançado, bem como do seu familiar. As ações, baseadas em valores defendidos no exercício da prática profissional, contemplam a integralidade a partir do envolvimento com o trabalho, respeito ao próximo e criação de vínculos, objetivando promover o conforto e qualidade de vida, conforme preceitos da atenção paliativa.

\section{[...] faço o curativo hoje e vou para casa pensando como} fazer melhor no dia seguinte, para dar o retorno e conforto, e fazer aquela pessoa se sentir melhor. E eu vou trabalhando assim. Crio vínculo com o paciente e com a família, não porque eu seja melhor que os meus colegas, é porque eu estou todos os dias aqui. (E-4).

Às vezes, o paciente não está muito interessado na dieta, ele quer que você toque, faça uma massagem de conforto, um carinho, passe um algodão úmido no olho dele [...]. É muito interessante. Chegar com um sorriso [...]. Mas eu também respeito muito o silêncio dele. Quando eles estão quietos, diminuo até o meu tom de voz quando chego ao quarto. (TE-8).

Os enfermeiros ressaltam a importância de o trabalho ser desenvolvido em tempo hábil, a partir da valorização das questões psicossociais, emocionais, espirituais e culturais, que podem estar envolvidas nos processos da doença e do morrer vivenciados pela pessoa e seus familiares.

$\mathrm{Na}$ visita leito a leito já vou estabelecendo as prioridades, vejo os pacientes que tem curativo, os que não estão bem emocionalmente, e nestes casos eu já sinalizo aos demais profissionais, porque como eu sou a referência para a equipe [...]. Eu dou o suporte emocional que posso na hora, e assim que eu puder já encaminho para quem é de direito. É isso! [...] muitas vezes eu não posso dizer que volto depois, porque não há tempo para isso. (E-5).

E eu pergunto: "- Seu José, o senhor está com dor? De 0 a 10, como está essa dor? O senhor está triste? Hoje eu fiz essa pergunta para um paciente e ele respondeu: "Nesse ai você pode colocar dez". E, então, eu já falei com a psicóloga, e decidimos encaminhá-lo para o setor onde desenvolvemos atividades lúdicas. Quando ele voltar daqui a pouco vou perguntar se a tristeza melhorou (E-4).

É válido ressaltar que, em prol da integralidade do cuidado, a equipe precisa atentar para as necessidades do familiar, que adoece junto e enfrenta dificuldades. A atenção à família nesta circunstância é aplicar a integralidade, considerando sua noção a partir das práticas profissionais.

Porque o paciente está doente, mas quando existe uma estrutura em volta dele, toda ela fica doente também. Sejam os familiares, os amigos, quem estiver ali com aquele paciente [...]. E a gente tem que identificar as necessidades, e enquanto enfermeiro fazer o que pode, em questão de orientação, de auxiliar em algum procedimento e em alguma coisa que ele precise, e encaminhar para os outros profissionais (E-2).

\section{- trabalho em equipe com premissa da integralidade do cuidado}

Esta unidade temática abrange a interação entre os membros da equipe de enfermagem e de saúde para o atendimento integral das necessidades de cuidado da pessoa hospitalizada e do seu familiar. Considera-se a postura profissional fundamental para a aplicação da integralidade, assim como o modo de trabalho no âmbito da equipe, bem como de organização dos processos relacionados.

[...] Eu me vejo como alguém que ajuda a equipe interdisciplinar, estando envolvido no trabalho, passando o máximo de informações possíveis para ter uma comunicação entre a equipe, que é muito importante para a qualidade do cuidado prestado ao paciente. (TE9).

O trabalho no âmbito da equipe de enfermagem é marcado pela relação de interdependência e valorização da comunicação. A relação é estabelecida na busca do apoio mútuo, diante da demanda de trabalho diária, 
ampliando as possibilidades de atendimento das necessidades da pessoa e dos seus familiares.

É uma relação de equipe mesmo. Aquele tempo em que o enfermeiro está gerenciando de uma maneira mais burocrática, no papel, nós estamos lá diretamente com o paciente identificando os problemas e passamos isso para ele [...]. A presença do enfermeiro é fundamental, porque acontecem situações que são difíceis de serem resolvidas. (TE-8)

E os enfermeiros também estabelecem comunicação entre si na organização das atividades, incluindo as gerenciais e as assistenciais, considerando a presença do plantonista e do diarista na maioria das enfermarias no plantão diurno.

A interação entre os profissionais tem como objetivo a formação de uma rede de atendimento, em prol da dinâmica do trabalho e da exequibilidade do cuidado. E a equipe de enfermagem tem grande participação e contribuição.

[...] o banho no leito de uma paciente com múltiplas fraturas foi realizado pela enfermagem junto com a fisioterapeuta. E não estava só o técnico, estávamos eu como enfermeira e o fisioterapeuta também. Teve outro caso, que durante o banho um paciente apresentou sangramento, e a médica entrou no banho junto com a gente. Então aqui temos interdisciplinaridade, existe isso, e o enfermeiro é o gerente. (E-2).

Eu me vejo como uma pessoa importante no trabalho. O técnico de enfermagem passa a maior parte do tempo com o paciente, então, acho que é minha obrigação, desde a lâmpada que não está funcionando até a administração do medicamento, ou qualquer outra coisa, por exemplo, que seja função do enfermeiro, eu tenho que sinalizar para ele. Se for uma coisa relacionada com a dieta, se o paciente estiver vomitando, eu tenho que sinalizar para a nutricionista, tenho que sinalizar para o médico se não tiver nada prescrito. Então, se eu que tenho mais contato com o paciente não fizer esse trabalho não vai ter o cuidado paliativo. Para mim, não existe cuidado paliativo sem técnico de enfermagem, ou sem qualquer outro profissional da equipe. Toda a equipe é importante. Até os funcionários da limpeza são importantes, pois se o quarto estiver com odor, se não estiver limpo, o paciente não ficará confortável. (TE-10).

É destacada a importância do profissional enfermeiro diarista no estabelecimento da comunicação eficaz, a favor da integralidade, uma vez que detém maior domínio dos acontecimentos diários, tendo maior subsídio para acompanhar os casos. O enfermeiro diarista é tido como elemento referência para a equipe, pessoas hospitalizadas e familiares, favorecendo a continuidade do cuidado. Além disso, neste aspecto a enfermagem se fortalece pelo fato de estar presente 24 horas no setor.

Eu vejo que tem muito mais facilidade quando a gente é diarista, para ver se aquela demanda do dia anterior foi resolvida ou não. Então, a gente consegue perceber se houve resolutividade do problema. Você consegue ver a sequência do cuidado mais facilmente [...]. Na maioria das vezes é o enfermeiro o elemento principal da equipe multidisciplinar [...]. Acho que o enfermeiro é primordial na equipe. Ele é que faz o elo realmente. Ele que fica mais tempo com o doente, está mais próximo (E-3).

A rotina do trabalho em equipe começa com a prática chamada de round, onde todos repassam as informações de cada caso e direcionam condutas, de forma conjunta.

A comunicação estabelecida é essencial na conjunção de valores expressos na forma como os profissionais buscam, juntos, atender as necessidades da pessoa e seus familiares.

Todos conversam pela manhã na passagem leito a leito dos pacientes, conversando com os mesmos e com seus familiares. E eles falam que aqui é diferente porque conversamos, tocamos o paciente. (E-4).

Em geral, o trabalho da equipe de enfermagem se desenvolve pautado no olhar ampliado acerca das necessidades da pessoa e dos seus familiares, o que favorece a integralidade do cuidado.

Eu tento fazer o que eu, enquanto enfermeira e pessoa, posso para ajudar no conforto emocional. De estar ali, de conversar, de ver o que a gente pode fazer para fortalecer os laços familiares. Nós enquanto enfermeiras podemos conversar com a família, e também falar com os outros profissionais que podem estar envolvidos nisso. $E$ a gente enquanto equipe, gerenciar essa questão. (E-2)

E eu não sou de dar só um "jeitinho", eu tenho um olhar que quando eu chego para trocar uma fralda, ou administrar uma medicação, eu já converso com o familiar, com o paciente, já identifico algumas situações 
[...]. Eu não chego lá e só coloco a medicação e troco a fralda. Eu evito perguntas que te dão respostas rápidas. Eu não pergunto: "_Está tudo bem?". Dessa forma eu não estou dando a ele momento nenhum de resposta. Daí eu pergunto: "_Está sentindo alguma coisa?" ou "_ Como o senhor está nesse momento?" (TE-8)

\section{DISCUSSÃO}

Os meios para a integralidade do cuidado à pessoa com câncer em processo de finitude podem ser compreendidos a partir do reconhecimento de suas múltiplas dimensões e das relações entre elas, bem como de sua individualidade ${ }^{(4,15)}$.

Dessa forma, o enfermeiro, na ótica do cuidado ampliado, deve considerar a pessoa em sua totalidade, reconhecendo que sua singularidade é uma característica comum a todas as pessoas, sendo, entretanto, o elemento que a diferencia das demais ${ }^{(15)}$.

No cuidado ao fim da vida, a dialógica vida/morte desperta a consciência do tempo e a necessidade de enfrentamento da realidade. $E$, paradoxalmente, as situações se entrelaçam no cotidiano de trabalho da enfermagem, avivando a necessidade de o cuidado ser estruturado na criação de vínculo, no respeito ao próximo e na ética, a partir da compreensão da interdependência das unidades dialógicas coletivo/indivíduo e pessoa/família.

Nesse sentido, ressalta-se a preocupação dos profissionais da equipe de enfermagem em considerar o familiar com uma unidade de cuidado, o que vai ao encontro da contextualização, valorizando a estrutura social na qual a pessoa faz parte. Destacaram-se como estratégias para o cuidado a comunicação, a criação de vínculos e as ações multidisciplinares para o atendimento das necessidades da pessoa e dos seus familiares, fortalecendo os laços entre os mesmos. A formação de redes entre os profissionais da área da saúde vai ao encontro dos objetivos da integralidade e da atenção paliativa ${ }^{(16)}$.

Evidencia-se a importância da comunicação e da interação entre as pessoas presentes no contexto. Na equipe de enfermagem a interação é marcada pelo apoio mútuo entre seus integrantes e convergência de esforços, o que se relaciona com o maior controle das situações, facilitando o atendimento das necessidades da pessoa e dos seus familiares. E para o bom desenvolvimento do trabalho grupal o enfermeiro precisa exercer a liderança de modo a estreitar as relações e participar as pessoas, sendo reconhecido pela equipe como fundamental para orientar as práticas e solucionar problemas difíceis, e como um profissional referência, em especial, quando diarista ${ }^{(17)}$.

Considerando o trabalho na perspectiva da interdisciplinaridade, a comunicação viabiliza a formação da rede de atendimento, a partir da troca de informações acerca dos casos gerenciados, em prol da integralidade. Ressalta-se a importância do sentimento de fazer parte da equipe, relacionado ao envolvimento, ao compromisso e à clareza das atribuições específicas e finalidades do trabalho(17). Tais elementos contribuem para a tomada de decisão articulada e para a própria organização do trabalho da equipe de enfermagem, capaz de refletir uma perspectiva colaboradora, integradora, e principalmente, satisfatória ${ }^{(18)}$.

Dessa forma, a integralidade é facultada pela interação e comunicação, ações elementares para o sucesso na sua aplicação e na prática interdisciplinar. Pode-se afirmar que é por meio do processo interativo que o enfermeiro realiza o cuidado. A interação, por si só, compreende um processo de percepção e comunicação entre pessoas a favor de um objetivo comum, e que mistura as particularidades inerentes às subjetividades das pessoas. A complexidade que emerge das interações múltiplas requer um olhar ampliado e postura flexível no reconhecimento dos diferentes fatos e fenômenos que acontecem no mesmo ambiente e que exercem influência direta nas ações de cuidado(19).

Ao encontro dos registros em literatura ${ }^{(19)}$, evidenciaram-se nos depoimentos dos sujeitos diversos instrumentos de interação, a favor do cuidado integral e da humanização, tais como: o emprego de atividades lúdicas, em especial, diante do sentimento de tristeza; o trabalho em equipe; a visita de enfermagem leito a leito, bem como em articulação com os demais profissionais da equipe de saúde durante o round; o estabelecimento de relações éticas e empáticas, sustentadas pelo escuta ativa e disponibilidade.

A aplicabilidade prática de tais instrumentos de interação demonstrou ser facilitada quando realizada pelo enfermeiro diarista, uma vez que este participa diariamente das histórias de vida e dos seus desdobramentos. Estes podem decorrer das mudanças impostas pela fase da doença e pela ameaça da morte, envolvendo sentimentos como medo, perda, culpa, dor, luto e alívio. Percebe-se que o enfermeiro diarista contribui para a continuidade do cuidado, sendo responsabilidade institucional pensar a distribuição dos recursos humanos e valorização da sua presença, como importante facilitador da integralidade e do trabalho em equipe. 
De acordo com os aspectos fundamentais para a humanização do cuidado, os preceitos da atenção paliativa e em respeito à autonomia da pessoa hospitalizada e dos seus familiares, os depoimentos encaminham para o reconhecimento da prática livre das relações de poder historicamente vigentes no ambiente hospitalar. Esta acontece a partir de abordagens diferenciadas e democráticas capazes de promover a participação ativa dos mesmos. Esta conduta, abrindo espaço para o diálogo e trocas entre o científico e o senso comum visa contribuir para a integralidade do cuidado, a partir de abordagens que vão além da sustentação do modelo dominante biomédico ${ }^{(20)}$.

Sendo assim, no contexto da atenção paliativa oncológica a integralidade do cuidado depende do empenho de todos os integrantes da equipe de saúde, sendo o tempo um grande aliado para o estabelecimento das relações de vínculo e confiança, consequentemente para o sucesso no cuidado. Contudo, em muitas situações, é preciso objetivar as ações e visar o atendimento das necessidades em tempo hábil, e nestes casos, diante de algumas peculiares da fase da doença ou limitações do ambiente de trabalho, a equipe de enfermagem precisa ter habilidades e competências múltiplas.

Dessa forma, destacam-se duas considerações importantes: a necessidade de estabelecimento de comunicação entre a oncologia e a atenção paliativa, de modo a favorecer o trabalho em concomitância, desde o momento do diagnóstico da doença que ameaça a vida. Esta consideração é reforçada perante dados que alertam sobre a relação entre o encaminhamento tardio no âmbito das diferentes propostas terapêuticas de cura e de paliação e o limitado acesso à integralidade ${ }^{(21)}$. A outra consideração decorre da necessidade da presença constante dos integrantes da equipe de saúde, visando o atendimento integral em tempo absoluto e não parcelado, incluindo os turnos noturnos de muitos serviços hospitalares, quando, em sua maioria, a equipe de enfermagem trabalha isoladamente ${ }^{(22)}$.

$\mathrm{Na}$ interdisciplinaridade é preciso comunicar os diferentes saberes científicos. $E$, na perspectiva do cuidado integral, enraizar a esfera antropossocial na esfera biológica, já que todo ser humano está inserido em um contexto, passível de problemas e consequências ${ }^{(15)}$.

Os ganhos para a qualidade do cuidado de enfermagem na atenção paliativa oncológica são fortalecidos, então, pela prática pautada no potencial do trabalho em equipe, objetivando a integralidade. Nesse sentido, ressalta-se a importância do planejamento e da organização da assistência por parte do enfermeiro, delineando ações que valorizam a subjetividade, o toque terapêutico, a escuta ativa e a promoção do conforto e da qualidade de vida. Desse modo, assistir o paciente com câncer vai além de uma prescrição de cuidados $^{(23)}$, em especial, na terminalidade, envolvendo sentimentos, atitudes, valores e crenças dos profissionais de enfermagem.

No que concerne às contribuições na assistência integral por parte do enfermeiro que assume a gerência do cuidado, com destaque para o enfermeiro diarista, além de representar um elo entre os demais profissionais da equipe, apontando demandas específicas, ressalta-se sua responsabilidade em assumir o compromisso de orientação e supervisão da equipe de enfermagem ${ }^{(23)}$. Assim, destaca-se nos depoimento dos técnicos de enfermagem a segurança em poder contar com este profissional, em especial, diante de conflitos difíceis de serem solucionados, muitas vezes relacionados com a possibilidade da morte, que se associam à complexidade dos problemas e às consequências da vida humana.

\section{CONCLUSÕES}

Neste estudo buscou-se identificar os indícios da integralidade do cuidado na prática dos profissionais da equipe de enfermagem que atuam nas enfermarias no contexto da atenção paliativa oncológica. Destacou-se o trabalho em equipe como premissa da integralidade do cuidado, valorizando a formação da rede de atendimento, considerando a dinâmica do trabalho e a exequibilidade do cuidado para o atendimento das necessidades da pessoa e do seu familiar. Nesta fase da vida, para a manutenção da qualidade dos dias de vida no processo de morrer por câncer, os objetivos do cuidado, ao encontro do preconizado pela OMS, abrangem a valorização e atendimento de tais necessidades que podem conjugar as dimensões física, psicológica, espiritual, social e cultural.

Assim, os indícios da integralidade do cuidado evidenciaram-se, dentre outros aspectos, na postura profissional e no modo de trabalho em equipe. Os enfermeiros e técnicos de enfermagem, a partir do sentimento de pertencer a uma equipe, do compromisso e comprometimento, valorizaram a troca de informações acerca dos casos gerenciados, e demonstraram possuir clareza das suas atribuições específicas e finalidades do trabalho, evidenciando valores congruentes com os preceitos da integralidade, bem como da atenção paliativa. No âmbito do trabalho em equipe, o enfermeiro 
demonstrou ser um grande articulador, diante da oportunidade de angariar subsídios para o reconhecimento das demandas, em especial, quando diarista, sendo o profissional de referência.

Deve-se considerar como impacto da pesquisa os aspectos a serem valorizados ao se pensar a formação da consciência da equipe de enfermagem como parte da equipe de saúde e em sua própria interdependência para atendimento integral na atenção paliativa. Assim, no que se refere às estratégias administrativas para dinâmica e organização do trabalho dos enfermeiros e técnicos de enfermagem, o incentivo à autovalorização e à autopercepção como elementos-chave da equipe compreende ação importante, o que deve ser favorecido por meio da disponibilidade de recursos para aplicação dos instrumentos de interação e da política

\section{REFERÊNCIAS}

1. Lei $n^{\circ} 8.080$ de 19 de setembro de 1990. Dispõe sobre as condições para promoção, proteção e recuperação da saúde, a organização e o funcionamento dos serviços correspondentes e dá outras providências. Diário Oficial da União (Brasília). 1990 Set 20.

2. Silva KB, Bezerra AFB, Tanaka OY. Direito à saúde e integralidade: uma discussão sobre os desafios e caminhos para a sua efetivação. Interface.

2012;16(40):249-260.

3. Andrade SR, Mello ALSF, Locks MTR, Mattia D, Hoeller $\mathrm{F}$, Erdmann AL. Melhores práticas na atenção básica à saúde e os sentidos da integralidade. Esc Anna Nery. 2013;17(4): 620-27.

4. Mariotti $\mathrm{H}$. Pensando diferente - para lidar com a complexidade, a incerteza e a ilusão. São Paulo: Atlas; 2010.

5. Gayeski ME, Parizoto GM, Guimarães GP, Erdmann AL, Meirelles BHS. Sistemas de organización de cuidados en enfermería: reflexiones sobre la complejidad del cuidado como práctica asistencial. Rev Cubana de Enfermer [Internet]. 2012; [cited 2014 Jun 26];28(1):48-62. Available from:

http://scielo.sld.cu/pdf/enf/v28n1/enf06112.pdf 6. World Health Organizations. National cancer control programmes: policies and managerial guidelines. 2 nd ed. Genebra: WHO; 2002.

7. Bakitas M, Lyons KD, Hegel MT, Ahles T. Oncologists' perspective on concurrent palliative care in National Cancer Institute-designated comprehensive cancer center. Palliat Support Care. 2013;11(5):415-23.

8. Van Beek K, Woitha K, Ahmed N, Menten J, Jaspers B, Engels $Y$, et al. Comparison of legislation, regulations and national health strategies for palliative care in seven European countries (Results from the Europall Research Group): a descriptive study. BMC Health Serv Res. 2013;13:275.

9. Thorne S, Oliffe JL, Stajduhar KI, Oglov V, Kim-Sing C, Hislop TG. Poor communication in cancer care: patient perspectives on what to do about it. Cancer Nursing. 2013;36(6):445-53. organizacional que apoie a prática interdisciplinar, por conseguinte, a integralidade.

$\mathrm{Na}$ perspectiva mais generalista sobre a temática alerta-se também sobre a emergência do tema e dos desafios que envolvem tanto a integralidade quanto a atenção paliativa oncológica, considerando, por exemplo, a luta contra o reducionismo e a fragmentação do cuidado, uma vez que na atenção paliativa oncológica, o atendimento integral visa atentar para as necessidades que vão além do aspecto físico. Esta realidade implica na necessidade de investimentos na pesquisa, a fim de contribuir para uma sociedade mais justa e solidária. Desse modo, a luta pela integralidade deve ser incentivada visando importantes transformações, na articulação dos seus sentidos, e na promoção da abertura ao diálogo.

10. Ferris FD, Bruera E, Cherny N, Cummings C, Currow $D$, Dudgeon $D$, et al. Palliative Cancer Care a Decade Later: accomplishments, the need, next steps - From the American Society of Clinical Oncology. J Clin Oncol. 2009;27(18):3052-58.

11. Cohen J, Deliens $L$, editors. A public health perspective on end of life care. $2^{\text {nd }}$ ed. United Kingdom: Oxford University Press; 2013.

12. Silva KS, Kruse MHL. Em defesa da sociedade: a invenção dos cuidados paliativos e os dispositivos de segurança. Texto Contexto Enferm. 2013; 22 (2): 51725.

13. Minayo MCS. O desafio do conhecimento: pesquisa qualitativa em saúde. $5^{\text {th }}$ ed. São Paulo: Hucitec; 2010. 14. Bardin L. Análise de conteúdo. Tradução de Luís Antero Reto e Augusto Pinheiro. Portugal: Edições 70; 2009.

15. Morin E. Ciência com consciência. 13 rd ed. Rio de Janeiro: Bertrand Brasil; 2010.

16. Hannon B, O'Reilly V, Bennett K, Breen K, Lawlor PG. Meeting the family: measuring effectiveness of family meetings in a specialist inpatient palliative care unit. Palliat Support Care. 2012;10(1):43-9.

17. Cardoso ASF, Dall'Agnol CM. Processo grupal: reflexões de uma equipe de enfermagem. Rev Esc Enferm USP. 2011;45(6):1412-8.

18. Matos E, Pires DEP, Gelbcke FL. Implicações da interdisciplinaridade na organização do trabalho da enfermagem: estudo em equipe de cuidados paliativos. Rev. Eletr. Enf. [Internet]. 2013 [cited 2013 jul 31];14(2):230-9. Available from: http://www.fen.ufg.br/revista/v14/n2/v14n2a02.htm 19. Lanzoni GMM, Lessmann JC, Sousa FGM, Erdmann $A L$, Meirelles BHS. Interações no ambiente de cuidado: explorando publicações de Enfermagem. Rev Bras Enferm. 2011;64(3):580-6.

20. Bonfada D, Cavalcante JRLP, Araujo DP, Guimarães J. A integralidade da atenção à saúde como eixo da organização tecnológica nos serviços. Ciênc saúde coletiva [Internet]. 2012 [cited 2013 abr 15];17(2):555- 
60. Available from:

http://www.scielo.br/pdf/csc/v17n2/a28v17n2.pdf

21. Hui D, Elsayem A, De La Cruz M, Berger A,

Zhukovsky DS, Palla $S$, et al. Availability and Integration

of Palliative Care at United States Cancer Centers. JAMA.

2010;303(11):1054-61.

22. Silva MM, Moreira MC, Leite JL, Erdmann AL. O

trabalho noturno da enfermagem no cuidado paliativo oncológico. Rev Latino-Am Enfermagem.

2013;21(1):773-779.

23. Silva RCV, Cruz EA. Planejamento da assistência de enfermagem ao paciente com câncer: reflexão teórica sobre as dimensões sociais. Esc Anna Nery.2011;15(1): 180-5.

Artigo recebido em 02/08/2013.

Aprovado para publicação em 17/06/2014.

Artigo publicado em 31/12/2014. 